\title{
12. Pflege und Unterstützung bei gesundheitlichen Einschränkungen: Welchen Beitrag leisten Personen in der zweiten Lebenshälfte für andere?
}

\author{
Daniela Klaus \& Clemens Tesch-Römer
}

\section{Kernaussagen}

Personen zwischen 40 und 85 Jahren unterstützen im Jahr 2014 häufiger und eine größere Anzahl gesundheitlich eingeschränkter Menschen als im Jahr 1996: Der Anteil derer, die andere unterstützen, ist von 12,3 Prozent auf 16,2 Prozent angestiegen. In demselben Zeitraum hat sich der Anteil derer, die mehr als eine Person unterstützen von 13,1 Prozent auf 23,8 Prozent erhöht.

Die für die am meisten unterstützte Person aufgewendete Zeit ist von 1996 zu 2014 zurückgegangen: Die aufgebrachte Zeit pro Woche hat sich von 19,8 Stunden (1996) auf 10,7 Stunden (2014) fast halbiert. Der Fokus der Unterstützung liegt zunehmend auf weniger aufwändigen Tätigkeiten wie Hilfe im Haushalt und seltener auf Pflegeaufgaben.

Der Großteil der Unterstützung wird im Jahr 2014 weiterhin innerhalb der Kernfamilie erbracht: Im Jahr 2014 unterstützen 17,0 Prozent ihre (Ehe-)Partnerin beziehungsweise ihren (Ehe-)Partner, 36,2 Prozent unterstützen ihre Mutter und 13,2 Prozent ihren Vater. Etwa ein Fünftel (21,1 Prozent) unterstützt nicht verwandte Personen.

Frauen vereinbaren häufiger Erwerbstätigkeit und Unterstützungsaufgaben als Männer und Hochgebildete häufiger als niedriger Gebildete. Im Jahr 2014 sind 13,9 Prozent der Frauen im Alter zwischen 40 und 65 Jahren erwerbstätig und übernehmen gleichzeitig Unterstützungsaufgaben (Männer: 9,6 Prozent). Unter Hochgebildeten beträgt der entsprechende Anteil 12,8 Prozent (niedrige Bildung: 5,1 Prozent).

\subsection{Einleitung}

In Deutschland leben derzeit etwa acht Millionen Menschen, die in ihren Aktivitäten des täglichen Lebens auf Dauer erheblich eingeschränkt sind. Laut der jüngsten Pflegestatistik (2013) sind davon 2,6 Millionen im Sinne der 1995 eingeführten sozialen Pflegeversicherung (SPV) leistungsberechtigt (Statistisches Bundesamt 2015b). Hierauf haben kranke oder behinderte Personen Anspruch, die bei Aktivitäten des täglichen Lebens wie Körperpflege,
Nahrungsaufnahme, in ihrer Mobilität oder ihrer hauswirtschaftlichen Versorgung in erheblichem Umfang und dauerhaft auf Hilfe angewiesen sind ( $\$ 14$ SGB XI). Schätzungen zufolge sind weitere 5,4 Millionen in diesem Sinne zwar nicht leistungsberechtigt, benötigen aber dennoch aufgrund gesundheitlicher Einschränkungen regelmäßige Unterstützung bei ihrer täglichen Versorgung (Geyer \& Schulz 2014). 
Unterstützung und Pflege werden mehrheitlich im häuslichen Bereich durch Familienangehörige, Nachbarinnen und Nachbarn, Freundinnen und Freunde oder ehrenamtlich Helfende erbracht. Nur teilweise werden sie durch professionelle Dienste ergänzt und noch seltener durch diese ersetzt - jedoch umso häufiger und umfangreicher, je schlechter die Gesundheit der hilfebedürftigen Person beziehungsweise je höher ihre Pflegestufe ist (Blinkert \& Klie 2006; Geyer \& Schulz 2014; Statistisches Bundesamt 2015b). Der Großteil derer, die aufgrund von Krankheit oder Behinderung auf die Hilfe anderer angewiesen sind, befindet sich bereits im hohen und sehr hohen Alter. Die zentralen Pflege- und Unterstützungspersonen älterer Menschen sind vor allem erwachsene Kinder und (Ehe-)Partnerinnen beziehungsweise (Ehe-) Partner (Haberkern \& Szydlik 2008; Klaus \& Tesch-Römer 2014; TNS Infratest Sozialforschung 2011).

Dabei übernehmen Frauen den größeren Teil der informellen Pflege sowie praktischen und zeitintensiven Unterstützung (Deindl, Brandt, \& Hank 2014; Haberkern, Schmid, \& Szydlik 2015; Khan 2014; Schmid, Brandt, \& Haberkern 2012; Wetzstein, Rommel, \& Lange 2015). Zudem deutet sich für niedrigere Bildungsgruppen eine größere Bereitschaft an, informelle Pflege zu leisten - anstatt Pflegedienste zu beauftragen (Blinkert \& Klie 2008; Heusinger 2007). Das mag einerseits an der schlechteren finanziellen Ausstattung von Personen mit niedrigem Bildungsniveau liegen, die es erschwert, professionelle Hilfe in Anspruch zu nehmen. Andererseits tragen sie - im Vergleich zu Hochgebildeten - geringere indirekte Kosten in Form von Einkommenseinbußen, wenn sie eine bestehende Erwerbstätigkeit reduzieren oder aufgeben, um eine andere Person zu pflegen.

Der hohe Stellenwert informeller Arrangements bei der Versorgung älterer Menschen spiegelt einerseits den weit verbreiteten Wunsch wider, im Alter so lange wie möglich zu Hause, im vertrautem Umfeld zu leben (Forschungsgesellschaft für Gerontologie e.V. 2006; Kuhlmey, Dräger, Winter, \& Beikirch 2010). Das geht einher mit einem in Deutschland ausgeprägten Familialismus, wonach die Hauptverantwortung für Unterstützung und Pflege Älterer mehrheitlich bei den Familienangehörigen - und weniger beim Staat - gesehen wird (Geerts \& van den Bosch 2012; Haberkern et al. 2015). Zum anderen resultiert die Dominanz informeller Arrangements aus den gesetzlichen Regelungen: So sieht beispielsweise die SPV keine vollständige Deckung des Pflegebedarfs durch professionelle Leistungen vor, vielmehr versteht sie sich ausdrücklich als Teilkasko-Leistung und damit insbesondere im ambulanten Bereich als Ergänzung zur Pflege durch Angehörige ( $\$ \$ 3$, 4 SGB XI). Diese Ausrichtung der Pflegepolitik ist Ausdruck des Subsidiaritätsprinzips, einer der wesentlichen Grundsätze des deutschen Sozialstaats: Danach sind staatliche Leistungen erst dann vorgesehen, wenn Familienangehörige sie nicht erbringen können.

Allerdings stößt das derzeitige System zur Versorgung kranker und hilfebedürftiger Menschen immer mehr an seine Grenzen, denn die Zahl älterer Menschen mit Unterstützungs- und Pflegebedarf wird in den kommenden Jahrzehnten (weiterhin) deutlich ansteigen - hauptsächlich als Folge der zunehmenden Hochaltrigkeit. Hinzu kommen die geburtenstarken Jahrgänge der zwischen Mitte der 1950er und Mitte der 1960er Geborenen (Babyboomer), die in den nächsten Jahren in Rente gehen werden und in absehbarer Zukunft ein Alter erreichen, in welchem sie zunehmend auf die Hilfe anderer angewiesen sein werden. Vorausberechnungen zufolge könnte allein die Zahl der Pflegeempfängerinnen und -empfänger auf bis zu 4,5 Millionen im Jahr 2050 steigen, was 6,5 Prozent der Gesamtbevölkerung entspräche (Statistische Ämter des Bundes und der Länder, 2010). Noch nicht berücksichtigt sind hierbei die älteren Menschen, die auch ohne Zuordnung in eine Pflegestufe im Alltagsleben Assistenz und Hilfe bei ihrer alltäglichen Lebensführung benötigen.

Die Herausforderungen, die sich aus dem wachsenden Bedarf für die Gesellschaft im Allgemeinen und die Familie im Speziellen ergeben, werden dadurch verstärkt, dass parallel zur steigenden Nachfrage insbesondere das familiale Unterstützungs- und Pflegepotenzial abnimmt. Bereits jetzt mindern gesunkene Kinderzahlen und gestiegene Wohnentfernungen zwischen 
Eltern und ihren erwachsenen Kindern (vgl. Kapitel 14) die Chancen auf (vor allem praktische) Unterstützung. Voraussichtlich wird sich die Zahl verfügbarer erwachsener Kinder und Partnerinnen beziehungsweise Partner in zukünftigen Generationen älterer Menschen weiter reduzieren (Dudel 2015; Haberkern \& Szydlik 2008). Ursächlich hierfür sind die zunehmende Kinderlosigkeit, die sich schon im Jahr 2014 in den jüngsten Kohorten des Deutschen Alterssurveys (DEAS) zeigt (vgl. Kapitel 14) sowie die anhaltend wachsende Instabilität und DeInstitutionalisierung von Partnerschaften, für die sich ebenfalls erste Hinweise in den jüngsten DEAS-Kohorten finden (vgl. Kapitel 13).

Die genannten demografischen Trends implizieren nicht nur eine Veränderung der Unterstützungs- und Pflegequote, sondern auch eine Veränderung in der personellen Zusammensetzung der Gruppe der Hilfeleistenden sowie der für sie daraus resultierenden Belastung. Theoretischen Modellen zur Unterstützung folgend sind erwachsene Kinder und Partnerinnen beziehungsweise Partner zwar die bevorzugten Unterstützungspersonen (Litwak 1985), weil sich Eltern-Kind-Beziehungen und Partnerschaften häufig durch eine hohe emotionale und meist auch räumliche Nähe auszeichnen und oft auf eine lange Beziehungs- und Austauschgeschichte zurückblicken. Diese Faktoren fördern die Motivation, im Bedarfsfall Hilfe zu leisten (u. a. Klaus 2009). Dennoch können Kinder und Partnerinnen beziehungsweise Partner durch andere Unterstützungspersonen ergänzt oder ersetzt werden, sollten sie nicht existieren oder beispielsweise aufgrund großer räumlicher Entfernung schlecht verfügbar sein (Cantor 1979). Demzufolge ist ein Bedeutungszuwachs der erweiterten Familie sowie nicht-familialer Beziehungspartner bei der Erbringung von Versorgungs- und Pflegeleistungen denkbar. Alternativ könnte sich mehr Arbeit auf weniger Personen verteilen, was einen Anstieg der Belastung für die Hilfeleistenden und Pflegenden mit sich bringen würde.

Neben den Veränderungen der Familienstrukturen hat auch die gestiegene Erwerbstätigkeit die Bedingungen informeller Pflege und Unterstützung maßgeblich beeinflusst. Immer mehr Pflege- und Hilfeleistende sind erwerbstätig. Zwischen 2001 und 2012 ist unter den informell Pflegenden im erwerbsfähigen Alter (unter 65 Jahren) der Anteil der Erwerbstätigen von 53 auf 66 Prozent angestiegen - wobei ein Großteil in Teilzeit erwerbstätig ist (Geyer \& Schulz 2014; ähnliche Zahlen bezogen auf die Hauptpflegeperson von Älteren mit Pflegestufe: TNS Infratest Sozialforschung 2011). Da sich vor allem Frauen verstärkt am Arbeitsmarkt beteiligen, sie aber den Großteil der Pflege und Unterstützung von Angehörigen tragen, wächst auch hier - analog zur Kinderbetreuung - der Handlungsbedarf zur Erleichterung der Vereinbarkeit zusehends. So ist die Schaffung entsprechender Rahmenbedingungen notwendig, die es erlauben, kranke Angehörige trotz eigener Erwerbstätigkeit zu unterstützen oder zu pflegen.

Im deutschsprachigen Raum gibt es wenig Forschung zur Vereinbarkeit von Unterstützung beziehungsweise Pflege und Erwerbstätigkeit, und entsprechend weiß man bislang nicht viel über die Situation Erwerbstätiger, die für andere Versorgungsverantwortung übernehmen. Offenbar ist, dass berufliche Verpflichtungen nicht nur die Zeit für Pflege und Hilfe reduzieren, sondern auch die Flexibilität, sie zu erbringen einschränken. Und gerade die Pflege älterer Menschen ist mit Blick auf Umfang, Dauer und Zeiteinteilung schwer zu kalkulieren (Preuss 2014). Zumindest im Pflegefall können ergänzend Hilfen und Leistungen in Anspruch genommen werden, die sich für Pflegebedürftige aus der gesetzlichen Pflegeversicherung ableiten wie Tages- oder Kurzzeitpflege. Zudem wurden in den letzten Jahren das Pflegezeitgesetz (2008) und später die Familienpflegezeit (2011) erlassen, deren wesentliche Elemente eine Verbesserung der Vereinbarkeit durch mehr zeitliche Flexibilität sowie eine gewisse finanzielle Absicherung der Pflegenden vorsehen. Von diesen gesetzlichen Möglichkeiten haben allerdings bislang nur sehr wenige Gebrauch gemacht (Isfort 2013; Stüben \& von Schwanenflügel 2015) - einerseits weil diese Maßnahmen oft nicht bekannt sind und andererseits, weil sie häufig nicht ausreichen, um die notwendige Flexibilität und finanzielle Absicherung zu gewährleisten. Da jedoch auch ein nicht unerheblicher 
Unterstützungsbedarf Älterer bereits unterhalb der Schwelle zur gesetzlich definierten Pflegebedürftigkeit besteht und dieser Bereich noch viel weniger im Fokus sozialpolitischer Maßnahmen steht, ist von einer Situation auszugehen, die sich für die Betroffenen sehr schwierig gestaltet. In diesem Fall besteht oft nur die Möglichkeit, weitere Quellen informeller und ambulanter Unterstützung zu mobilisieren wie Verwandte, Nachbarn, bezahlte Dienstleistungen (Haushaltshilfen, ,Essen auf Rädern` etc.) oder auch ehrenamtliche Betreuung. Das setzt allerdings die Verfügbarkeit entsprechender Unterstützungspersonen voraus beziehungsweise finanzielle Mittel zur Inanspruchnahme bezahlter Hilfen.

Die bestehende Vereinbarkeitsproblematik könnte sich zudem durch den mit dem Aufschub der Familiengründung vergrößerten Generationenabstand verschärfen. Seit einigen Jahren wird die sogenannte ,Sandwich-Generation' als mittlere Generation in der Familie diskutiert, die zunehmender Mehrfachbelastung ausgesetzt ist. Das sei vor allem Folge demografischer Verschiebungen, die dazu führen, dass im mittleren Erwachsenenalter Erwerbstätigkeit immer häufiger mit Pflegetätigkeiten für ältere Generationen und Betreuungsaufgaben für jüngere Generationen zusammenfällt. Auch wenn diese Konstellation oft als sehr problembehaftet diskutiert wird, war sie zumindest noch vor einigen Jahren empirisch eine Ausnahme. Eine besondere Belastung für die betroffene $\mathrm{Ge}$ neration konnte bislang auch nicht belegt werden (Künemund 2002). Die genannten Trends implizieren allerdings einen Anstieg von Doppel- oder Mehrfachverantwortungen, wovon bestimmte Bevölkerungsgruppen stärker betroffen sein könnten als andere (Preuss 2014): Frauen aufgrund der nach wie vor verbreiteten klassischen geschlechtsspezifischen Arbeitsteilung und Höhergebildete aufgrund ihrer hohen Erwerbsorientierung, sofern deren bessere finanziellen Ausstattung nicht zu einer verstärkten Inanspruchnahme bezahlter (professioneller) Dienste führt.

Das vorliegende Kapitel beschäftigt sich mit Unterstützung und Pflege, die Personen in der zweiten Lebenshälfte für andere erbringen, die krank oder pflegebedürftig sind. Es werden folgende Fragen beantwortet:

1. Ist der Anteil derer, die andere aufgrund deren gesundheitlicher Beeinträchtigungen unterstützen, in den letzten Jahren zurückgegangen oder angestiegen? Zeichnen sich geschlechts-, alters- oder bildungsspezifische Unterschiede oder Entwicklungen ab?

2. Beteiligen sich 2014 neben der Kernfamilie verstärkt andere Familienmitglieder (wie Enkel und Geschwister), Verwandte (wie Nichten oder Neffen) oder auch Freundinnen und Freunde oder Nachbarinnen und Nachbarn an der Unterstützung?

3. Verteilt sich 2014 mehr Arbeit auf weniger Schultern und hat in dessen Folge die Belastung zugenommen?

4. Ist der Anteil derer, die neben ihrer Erwerbstätigkeit eine andere Person unterstützen und zudem Verantwortung für minderjährige Kinder haben angestiegen? Gibt es bestimmte Bevölkerungsgruppen, die hiervon besonders betroffen sind? 


\subsection{Datengrundlage ${ }^{1}$}

Daten. Zur Beantwortung der Fragen wurden die Querschnittsstichproben des Deutschen Alterssurveys (DEAS) der Jahre 1996, 2002, 2008 und 2014 verwendet (vgl. Kapitel 2). Für die meisten berichteten Indikatoren liegen Informationen zu allen Messzeitpunkten vor, vereinzelt aber auch erst ab 2002. Sofern nicht anders ausgewiesen, wurden Personen im Alter zwischen 40 und 85 Jahren betrachtet. Die Daten, die Grundlage für die folgenden Indikatoren bilden, wurden durchweg im Rahmen mündlicher Interviews erhoben.

Unterstützerquote. Seit 1996 wird in nahezu unveränderter Form erfragt, ob der oder die Befragte andere Personen aufgrund deren schlechten Gesundheitszustands privat oder ehrenamtlich betreut oder pflegt oder regelmäßig Hilfe leistet. Aufgrund leichter Modifikationen der Frage ist für die Jahre 1996 bis 2008 eine leichte Überschätzung der Prävalenzraten von Unterstützenden zu erwarten: So wurde 1996 nicht nur nach gesundheitsbedingter Hilfe und Pflege gefragt. In den Jahren 2002 und 2008 wurden nur Befragte berücksichtigt, die selbst nicht auf Hilfe oder Pflege angewiesen sind - wohingegen die Frage im Jahr 2014 allen Befragungspersonen unabhängig von deren eigenem Gesundheitszustand gestellt wurde.

Unterstützungspersonen. Die Befragten werden gebeten, alle Personen aufzulisten, die sie unterstützen. Für alle genannten Personen wird die Art der Beziehung erfasst. Dabei wird zwischen verschiedenen Verwandtschaftsgraden (wie Kind, Mutter, Vater, Schwiegermutter, Schwiegervater, Bruder, Schwester), Partnerschaft beziehungsweise Ehebeziehung sowie diversen Beziehungen zu Nicht-Verwandten unterschieden. Für die Auswertungen wurden folgende Empfänger(gruppen) betrachtet beziehungsweise zusammengefasst: (Ehe-) Partnerinnen und (Ehe-)Partner, Mutter, Vater, Schwiegereltern, Mitglieder der erweiter-

1 Die Daten des DEAS können für wissenschaftliche Zwecke kostenlos beim Forschungsdatenzentrum des DZA (www.fdz-dza.de) bezogen werden. ten Familie, Nicht-Verwandte (Freunde und Freundinnen, Bekannte, Arbeitskollegen und Arbeitskolleginnen).

Belastung. Seit dem Erhebungsjahr 2002 wird die Belastung durch alle erbrachten Unterstützungs- und Pflegeleistungen abgefragt. Dazu steht den Befragten eine vierstufige Antwortskala zur Verfügung, die von 1 , gar nicht belastet' bis 4 ,sehr stark belastet' reicht. Auf dieser Basis wurde folgende Zuordnung der Befragten in eine der beiden Gruppen vorgenommen: Solche, die sich (gar) nicht belastet fühlen (Antwortcodes 1 und 2) und diejenigen, die sich (sehr) stark belastet fühlen (Antwortcodes 3 und 4).

Art der Unterstützung, Pflege und Umfang. Nur für die Person, die laut Einschätzung der Befragten am meisten von ihnen unterstützt wird, wird die gegebene Unterstützung näher qualifiziert. Ab 2002 werden drei Leistungsarten unterschieden: Hilfe im Haushalt, Betreuung oder Begleitung und Pflege. Darüber hinaus wird der Umfang an wöchentlich für diese Aufgaben aufgebrachten Stunden erfasst - diese Information liegt ab 1996 vor.

Doppel- und Mehrfachverantwortungen. Um die Entwicklung von Mehrfachverantwortlichkeiten nachzuzeichnen, wurden für Personen im erwerbsfähigen Alter (40 bis 65 Jahre) Indikatoren gebildet, die angeben, ob zusätzlich zur Übernahme von Unterstützungsleistungen für andere (a) noch einer Erwerbstätigkeit nachgegangen wird (Vollzeit, Teilzeit, unregelmäßig oder geringfügig) und ob (b) neben Unterstützungs- und Erwerbstätigkeit Verantwortung für mindestens ein minderjähriges Kind im Haushalt zu tragen ist.

Gruppierungsvariablen. Es wurden Geschlechtsunterschiede untersucht. Zur Prüfung von Altersunterschieden wurden die folgenden Sechs-Jahres-Altersgruppen verwendet: 42 bis 47 Jahre, 48 bis 53 Jahre, 54 bis 59 Jahre, 60 bis 65 Jahre, 66 bis 71 Jahre, 72 bis 77 Jahre und 78 bis 83 Jahre. Zur Untersuchung von Bildungsunterschieden wurden - basierend auf einer reduzierten ISCED-Klas- 
sifizierung - drei Bildungsgruppen gebildet: Personen mit niedriger, mittlerer und hoher Bildung (vgl. Kapitel 2).

Analyse. In den Abbildungen und im Text sind gewichtete (gruppenspezifische) prozen- tuale Verteilungen angegeben. Um Gruppenunterschiede auf Signifikanz zu testen, wurden in Abhängigkeit vom Skalenniveau logistische oder lineare Regressionen berechnet. Das genaue Vorgehen ist in Kapitel 2 beschrieben.

\subsection{Verbreitung der Unterstützung für andere und Entwicklung über die Zeit}

Zunächst stellt sich die Frage, wie verbreitet es unter Personen in der zweiten Lebenshälfte ist, andere, die gesundheitlich eingeschränkt sind, informell zu unterstützen oder zu pflegen. Zeichnet sich diesbezüglich ein Wandel seit der ersten Erhebung im Jahr 1996 ab und welche Bevölkerungsgruppen übernehmen diese Aufgabe vergleichsweise häufig?

\section{Personen zwischen 40 und 85 Jahren unterstützen im Jahr 2014 eine größere Anzahl gesundheitlich eingeschränkter Menschen als im Jahr 1996.}

Im Jahr 2014 erbringen 16,2 Prozent aller 40- bis 85-Jährigen für andere Personen gesundheitsbedingte Unterstützung oder Pflege, während die Anteile in den vorangegangenen Jahren signifikant geringer ausfielen (Abbildung 12-1): 12,3 Prozent (1996); 11,4 Prozent (2002), 12,7 Prozent (2008). Trotz des Rückgangs des familialen Hilfepotenzials ist diese Quote 2014 gegenüber allen vorherigen Befragungsjahren signifikant angestiegen. Dabei ist davon auszugehen, dass dieser Anstieg angesichts oben genannter Gründe sogar noch unterschätzt sein könnte.

Der Anstieg dürfte Ausdruck eines wachsenden Bedarfes nach informeller Unterstützung und Pflege sein, den Personen in der zweiten Lebenshälfte offenbar - trotz Verkleinerung des engen Familienverbandes - decken. Die gestiegene Nachfrage nach Unterstützung und Pflege ist in erster Linie Folge der Alterung der Gesellschaft: Denn auch wenn Menschen immer länger gesund bleiben, nimmt der Anteil Unterstützungs- und Pflegebedürftiger zu. So belegt die jüngste Pflegestatistik einen Anstieg der Pflegebedürftigen in der Bevölkerung zwischen 1999 und 2013 von 2,5 auf 3,3 Prozent (Statistisches Bundesamt 2015b). Diese Zunahme ist auf den wachsenden Anteil alter und sehr alter Menschen zurückzuführen. Zugleich deckt die jüngste Pflegestatistik auf, dass der um den Jahrtausendwechsel noch deutliche Anstieg vollstationärer Betreuung in Heimen in den letzten Jahren abgeflacht ist. $\mathrm{Ob}$ die rückläufige Nutzung institutioneller Betreuungseinrichtungen auf deren unzureichende Verfügbarkeit zurückgeht oder auf Finanzierungsprobleme seitens der Betroffenen verweist, muss hier offen bleiben. Möglicherweise haben auch finanzielle Anreize wie das Pflegegeld dazu beigetragen, dass Pflege häufiger von Angehörigen übernommen wird.

\section{Unterstützung hat sich in ein höheres Alter verschoben.}

Wie die Abbildung 12-1 ebenfalls illustriert, wird der jüngste Anstieg des Anteils Unterstützungsleistender vor allem von den Älteren getragen. Während die Quoten der unter 54-Jährigen stabil geblieben sind, zeigen sich bei den 54 - bis 83-Jährigen signifikante Anstiege zwischen vier und sieben Prozentpunkten (je nach betrachteter Altersgruppe). Diese Verlagerung von Pflege und Unterstützung in ein höheres Alter spiegelt einerseits einen zunehmenden Versorgungsbedarf vor allem im hohen und sehr hohen Alter wider, der sich daraus ergibt, dass Menschen nicht nur länger leben, sondern auch länger gesund bleiben 
- zumindest im sogenannten ,dritten Lebensalter' (bis etwa 80/85 Jahre). Andererseits ist ein längeres Leben in Gesundheit auch auf Seiten der (potenziell) Hilfeleistenden Voraussetzung dafür, trotz eigenen fortgeschrittenen Alters Unterstützung und Pflege leisten zu können.

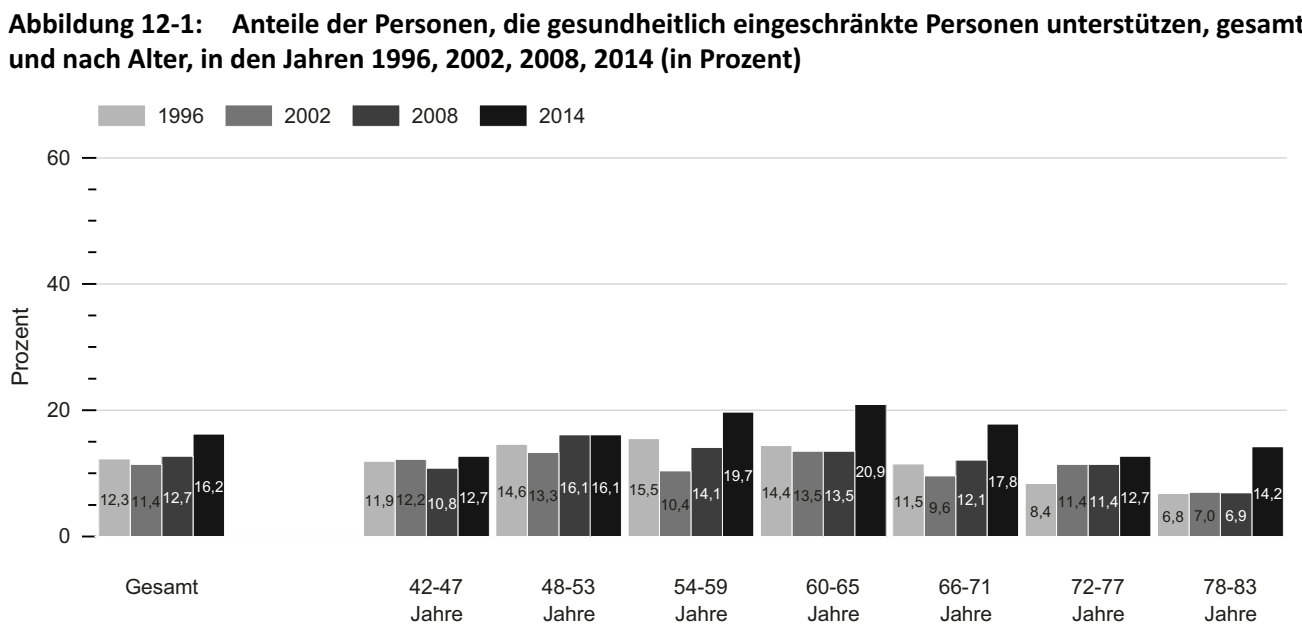

$\overline{\text { Quelle: DEAS } 1996}(n=4.835), 2002(n=2.999), 2008(n=5.860), 2014,(n=5.993)$, gewichtet, gerundete Angaben; $(p<, 05)$.

Signifikante Unterschiede zwischen 1996 und 2014 für die gesamte Stichprobe sowie für die Altersgruppen ab 54 Jahren. Kein signifikanter Unterschied zwischen 1996 und 2014 für die 42- bis 47-Jährigen und die 48- bis 53-Jährigen.

\section{Frauen unterstützen nach wie vor häufiger als Männer.}

Frauen übernehmen zu allen Zeitpunkten zwischen 1996 und 2014 häufiger Versorgungstätigkeiten im Krankheits- oder Pflegefall für andere als Männer. Im Jahr 2014 beträgt diese
Quote bei den Frauen 18,8 Prozent gegenüber 13,3 Prozent bei den Männern (Abbildung 122). Abgesehen von einem zwischenzeitlichen Rückgang bei den Männern findet bei beiden Geschlechtern ein Anstieg der Quote zwischen 1996 und 2014 statt. Der Unterschied zwischen den Geschlechtern hat sich über die Zeit hinweg nicht verändert. 


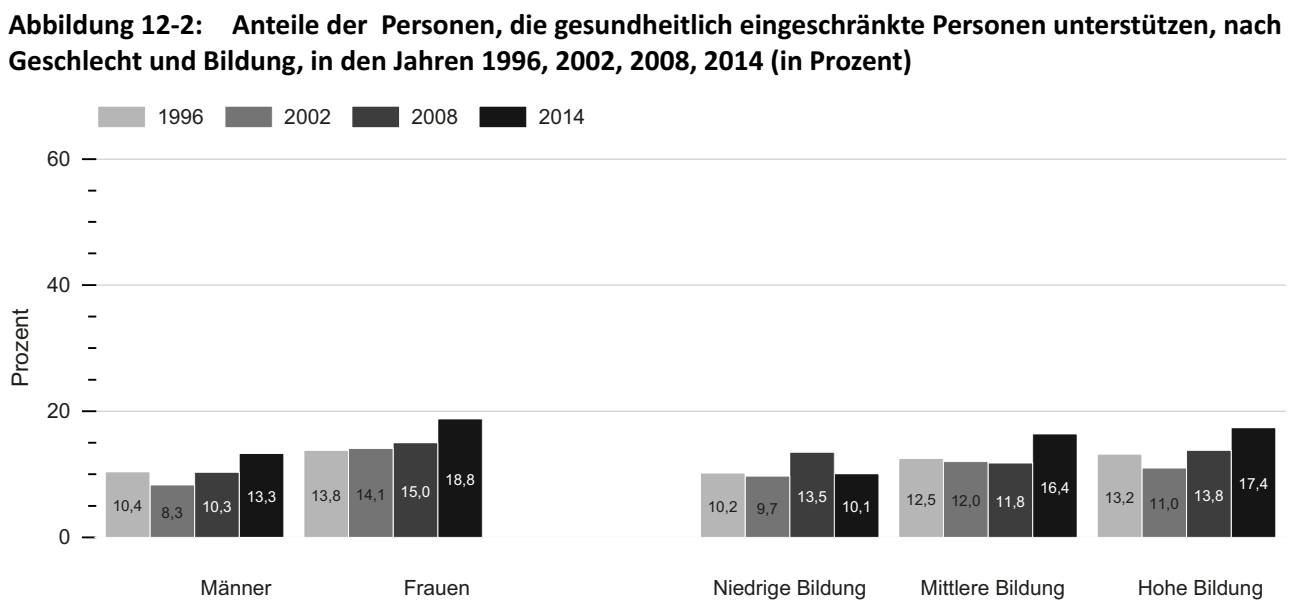

Quelle: DEAS 1996 ( $n=4.835), 2002$ ( $n=2.999), 2008(n=5.860), 2014,(n=5.993)$, gewichtet, gerundete Angaben; $(p<, 05)$.

Signifikante Unterschiede zwischen 1996 und 2014 für Männer und Frauen sowie Personen mit mittlerer und hoher Bildung. Keine signifikanten Unterschiede zwischen 1996 und 2014 für niedrige Bildung.

\section{Unterstützung hat vor allem unter Personen mit mittlerem und hohem Bildungsniveau zugenommen.}

Unterstützung erfolgt bildungsspezifisch - jedoch anders als es insbesondere der Forschungsstand zur Pflegeübernahme nahelegt. Die Abbildung 12-2 zeigt, dass Personen mit mittlerer und hoher Bildung durchweg häufiger Unterstützungsaufgaben für andere übernehmen als Personen mit niedriger Bildung. Beispielsweise beläuft sich die Quote unter den Personen

\subsection{Wer unterstützt wen?}

Wen pflegen und unterstützen Personen zwischen 40 und 85 Jahren, und ist es hier im Verlauf der letzten Jahre zu Verschiebungen gekommen? In Abbildung 12-3 sind alle von den Befragten genannten Empfängerinnen und Empfänger getrennt für die Erhebungsjahre dargestellt - teilweise in Gruppen zusammengefasst. mit mittlerem und hohem Bildungsniveau im Jahr 2014 auf 16,4 Prozent beziehungsweise 17,4 Prozent, bei denjenigen mit niedrigem Bildungsniveau liegt sie hingegen bei 10,1 Prozent. Signifikante Zuwächse zwischen 1996 und 2014 haben ebenfalls nur in den beiden höheren Bildungsgruppen stattgefunden - von 12,5 auf 16,4 Prozent beziehungsweise von 13,2 Prozent auf 17,4 Prozent - während der Wert in der niedrigen Bildungsgruppe nach einem zwischenzeitlichen Anstieg im Jahr 2008 wieder auf das Niveau von 1996 zurückgefallen ist: 10,2 Prozent beziehungsweise 10,1 Prozent.

\section{Der Großteil der Unterstützung wird im Jahr 2014 weiterhin innerhalb der Kernfamilie erbracht.}

Viele der 40- bis 85-Jährigen, die angeben, mindestens eine Person zu unterstützen oder $\mathrm{zu}$ pflegen, kümmern sich um ihre eigene Mutter: Im Jahr 2014 betrifft das mehr als ein Drittel 
(36,2 Prozent). Grundsätzlich liegt der Schwerpunkt informeller Unterstützung und Pflege im Einklang mit dem, was die Forschung bisher gezeigt hat - auf (Schwieger-)Eltern und (Ehe-) Partnerinnen beziehungsweise (Ehe-)Partnern. Daran hat sich seit 1996 wenig geändert: Weiterhin haben (Schwieger-)Kinder und (Ehe-) Partnerinnen beziehungsweise (Ehe-)Partner eine große Bedeutung beim Erbringen von Unterstützung und Pflege.

Zwar werden im Laufe der Jahre signifikant weniger Schwiegereltern genannt: Der Anteil ist von 21,0 Prozent im Jahr 1996 auf 13,8 Prozent im Jahr 2014 zurückgegangen. Andererseits werden im Jahr 2014 häufiger die eigenen Eltern versorgt (36,2 Prozent Mütter und 13,2 Prozent Väter) als 1996 (31,7 Prozent Mütter und 10,3 Prozent Väter). Auch die (ehe-)partnerschaftliche Pflege und Unterstützung hat deutlich zugenommen: Während 1996 etwa 10,1 Prozent eine (Ehe-)Partnerin beziehungsweise einen (Ehe-)Partner gepflegt oder unterstützt haben, sind es 2014 bereits 17,0 Prozent. Möglich ist das in erster Linie durch eine Veränderung der Sterblichkeit geworden. Die gestiegene Lebenserwartung hat dazu geführt, dass heute mehr Menschen mit ihren Eltern und ihrer (Ehe-)Partnerin oder ihrem (Ehe-)Partner gemeinsam alt werden können als noch vor einigen Jahren (vgl. Kapitel 13 und 14). Es gibt bislang keine Hinweise, dass sich veränderte Paarbildungs- und Paarauflösungsprozesse negativ auf die Versorgungssituation im Alter ausgewirkt haben: Trotz der gestiegenen Instabilität von Partnerschaften und Ehen und einem höheren Anteil Lediger in der zweiten Lebenshälfte (vgl. Kapitel 13) hat die partnerschaftliche Versorgung bislang nicht an Bedeutung eingebüßt. Möglicherweise ist eine solche rückläufige Entwicklung aber für zukünftige Kohorten zu beobachten.

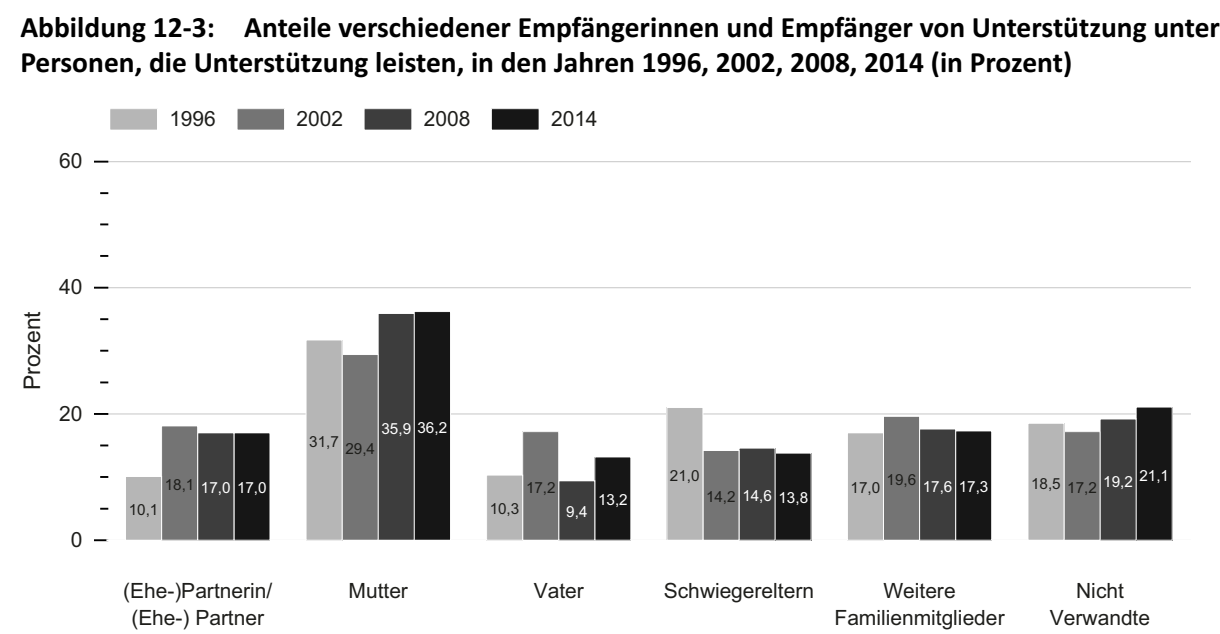

$\overline{\text { Quelle: DEAS } 1996}(n=561), 2002(n=325), 2008(n=713), 2014(n=958)$, Mehrfachnennung möglich, gewichtet, gerundete Angaben; $(p<; 05)$.

Signifikante Unterschiede zwischen 1996 und 2014 für (Ehe-)Partnerinnen und (Ehe-)Partner, Mütter, Väter und Schwiegereltern. Keine signifikanten Unterschiede zwischen 1996 und 2014 für weitere Familienmitglieder und Nicht-Verwandte.

Der leichte Anstieg der Unterstützung für NichtVerwandte von 18,5 Prozent auf 21,1 Prozent bleibt statistisch unbedeutsam ebenso wie sich der Anteil der Personen aus dem weiteren Familienkreis oder der Verwandtschaft mit rund 17
Prozent als stabil erweist (abgesehen von einem zwischenzeitlichen Anstieg im Jahr 2002 auf 19,6 Prozent).

Bilanzierend ist festzuhalten, dass sich - anders als vermutet - kein Trend abzeichnet, wo- 
nach sich Mitglieder der erweiterten Familie, Verwandte oder Nicht-Verwandte im Jahr 2014 häufiger an Versorgungsaufgaben im Krankheits- oder Pflegefall beteiligen würden als vor einigen Jahren. Im Gegenteil: Sogar stärker als 1996 dominiert bei der informellen Unterstützung die Kernfamilie.

\subsection{Unterstützungsumfang, Pflege und Belastung}

Mehr Menschen in der zweiten Lebenshälfte nehmen Unterstützungsaufgaben wahr. Zeigen sich auch Veränderungen in Umfang und Art der geleisteten Hilfe und damit einhergehender Belastung?

\section{Im Jahr 2014 werden mehr Personen unterstützt als in den Jahren zuvor.}

Nicht nur der Anteil derer, die andere unterstützen ist deutlich angestiegen, es wird auch immer häufiger mehr als nur eine Person versorgt: Waren es 1996 noch 13,1 Prozent, so hat sich dieser Anteil beinahe verdoppelt und liegt im Jahr 2014 bei 23,8 Prozent - ein signifikanter Anstieg (vgl. Tabelle A 12-3 im Anhang).

Abbildung 12-4: Häufigkeit verschiedener Arten der Unterstützung unter Personen, die Unterstützung leisten, in den Jahren 2002, 2008, 2014 (in Prozent)

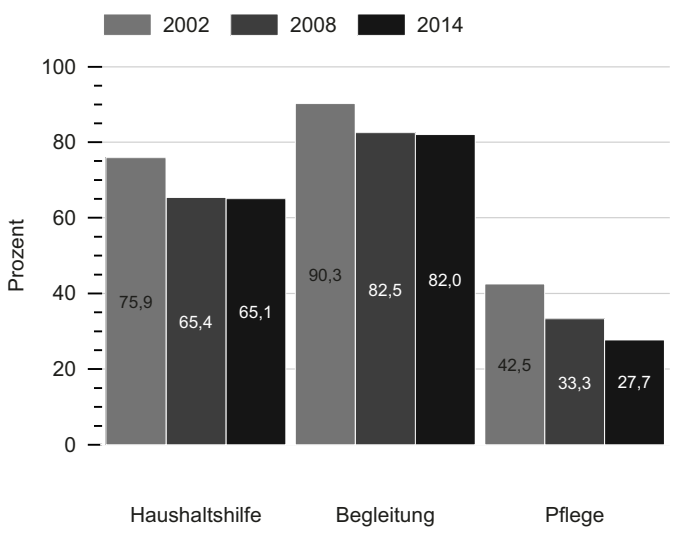

Quelle: DEAS $2002(n=313), 2008(n=720), 2014(n=966)$, Mehrfachnennung möglich, gewichtet, gerundete Angaben; $(p<, 05)$.

Signifikante Unterschiede zwischen 2002 und 2014 für alle drei Arten der Unterstützung.

Die für die am meisten unterstützte Person aufgewendete Zeit ist von 1996 zu 2014 zurückgegangen.

Parallel zur gestiegenen Zahl der unterstützten Personen hat sich der Umfang der geleisteten Aufgaben reduziert. So haben sich die für die am meisten unterstützte Person aufgewendeten Stun- den pro Woche nahezu halbiert: Ein signifikanter Rückgang von 19,8 Stunden (1996) auf 10,7 Stunden (2014) ist zu beobachten (vgl. Tabelle A 12-4 im Anhang). Dieser Befund spiegelt sich auch in der Abnahme der Breite der erbrachten Leistungen wider: Alle drei betrachteten Unterstützungsarten - Hilfe im Haushalt, Begleitung und Pflegeaufgaben - werden 2014 signifikant seltener erbracht als 
2002 (Abbildung 12-4). Zudem wird erkennbar, dass der Fokus gesundheitsbedingter informeller Unterstützung auf weniger aufwändigen Tätigkeiten liegt: So werden im Jahr 2014 Hilfe im Haushalt $(65,1$ Prozent) und Begleitung (82,0 Prozent) deutlich häufiger übernommen als Pflegeaufgaben (27,7 Prozent). Es lässt sich schlussfolgern: Personen in der zweiten Lebenshälfte unterstützen 2014 deutlich mehr Personen, die aufgrund gesundheitsbezogener Defizite auf Hilfe angewiesen sind, aber offenbar mit weniger Aufwand pro Person.

\section{Der Anteil derer, die Andere pflegen, liegt unverändert bei knapp fünf Prozent.}

Pflege stellt eine spezifische Art der Unterstützung dar, auf die gesundheitlich eingeschränkte Personen bei ihrer Alltagsbewältigung angewiesen sein können. Sie ist für die Pflegeleistenden eine oft körperlich sehr anstrengende und zeitintensive Aufgabe, die zum Teil auch Fachkenntnisse und (medizinisch-)technischer Hilfsmittel bedarf. Auch wenn die Ergebnisse belegen, dass unter denjenigen, die andere unterstützen, immer seltener Pflegeaufgaben übernommen werden (Abbildung 12-4), ist bezogen auf die Gesamtbevölkerung der 40- bis 85-Jährigen kein Rückgang der Pflegequote zu beobachten: 2002 haben 4,6 Prozent und 2014 haben 4,5 Prozent aller Personen in dieser Altersgruppe mindestens eine andere Person gepflegt (ohne Abbildung) ${ }^{2}$. Wird allerdings der im selben Zeitraum gestiegene Pflegebedarf in Rechnung gestellt, so deutet sich eine zunehmende Auslagerung dieser durchaus sehr voraussetzungsvollen Tätigkeit an. Es ist davon auszugehen, dass zur Pflege von Familienangehörigen verstärkt professionelle Hilfe wie die von ambulanten Pflegediensten hinzugezogen wird. Eine damit einhergehende zeitliche, körperliche und auch emotionale Entlastung könnte es den Unterstützungsleistenden ermöglichen, sich stärker auf weniger intensive Alltagsaufgaben zu konzentrieren. Möglicherweise liegt darin eine Ursache dafür, dass sie sich 2014 häufiger um mehr als eine Person kümmern beziehungsweise kümmern können.

\section{Unterstützungspersonen fühlen sich 2014 weniger häufig belastet als 2002.}

In diesem Sinne zeigen die Daten auch einen leichten Rückgang der subjektiv empfundenen Belastung durch die Übernahme von Unterstützungsaufgaben: Während sich im Jahr 2002 noch 38,6 Prozent (sehr) stark belastet fühlten, betrifft das 2014 einen signifikant geringeren Anteil von 31,7 Prozent (vgl. Tabelle A 12-6 im Anhang).

2 Verglichen mit der 2012 durchgeführten Studie,Gesundheit in Deutschland aktuell' (Wetzstein, Rommel, \& Lange 2015) ist davon auszugehen, dass der Anteil der Pflegenden im DEAS unterschätzt wird.

\subsection{Doppel- und Mehrfachverantwortungen}

Angesicht der steigenden Erwerbsbeteiligung im späten Erwerbsalter (vgl. Kapitel 3) sowie des hier berichteten, wachsenden Anteils von Personen in der zweiten Lebenshälfte, die andere unterstützen, ist davon auszugehen, dass eine immer größere Anzahl von Personen im erwerbsfähigen Alter vor der Aufgabe steht, beide Aufgaben miteinander zu vereinbaren. Lässt sich diese Vermutung bestätigen?

\section{Erwerbstätigkeit wird zunehmend mit Unterstützungsaufgaben vereinbart.}

Die Ergebnisse in Abbildung 12-5 bestätigen: Der Anteil der Personen im erwerbsfähigen Alter zwischen 40 und 65 Jahren, die informell Unterstützung leisten und zugleich erwerbstätig sind, ist von 7,0 Prozent (1996) auf 11,8 Prozent (2014) signifikant angestiegen. Parallel dazu ist der Anteil der ausschließlich Unterstützung Leistenden von 6,5 Prozent (1996) auf 5,1 Prozent (2014) leicht gesunken. 


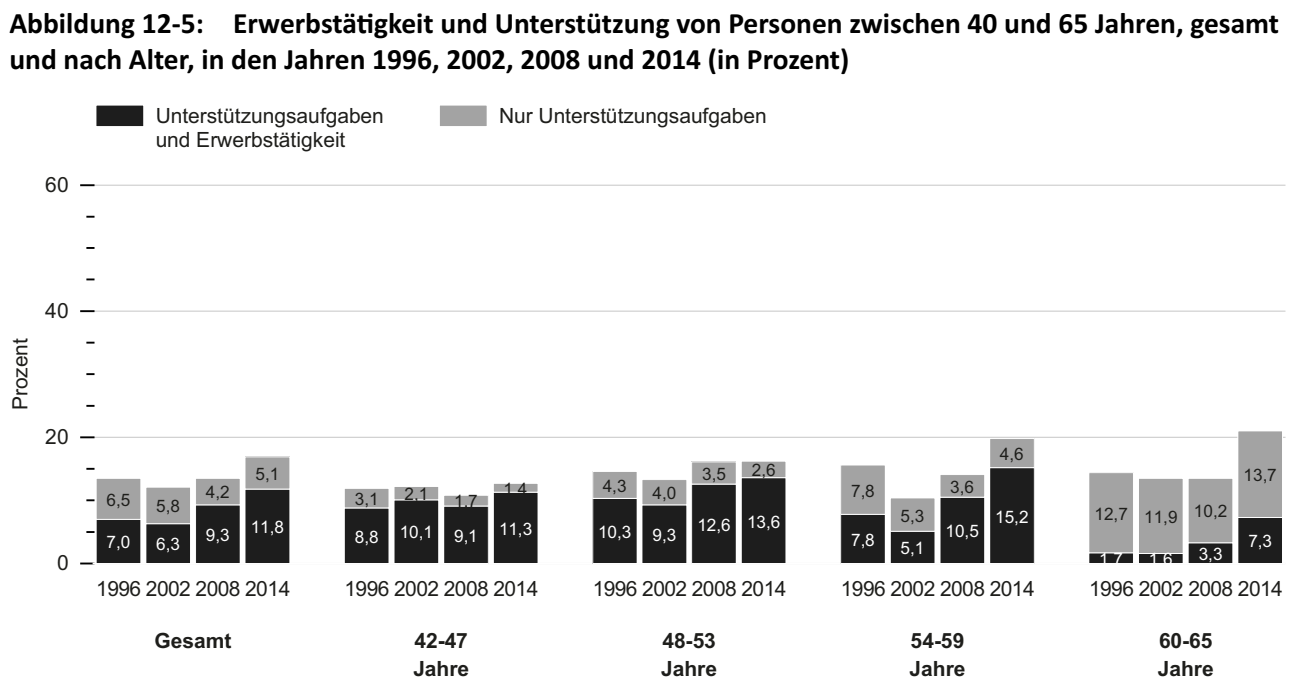

$\overline{\text { Quelle: DEAS } 1996}(n=3.132), 2002(n=1.834), 2008(n=3.480), 2014(n=3.526)$, gewichtet, gerundete Angaben; $(p<.05)$.

Signifikante Unterschiede zwischen 1996 und 2014 für die gesamte Stichprobe, sowie für alle Altersgruppen.

Die Gleichzeitigkeit beider Aufgaben - Erwerbstätigkeit und Unterstützungsleistung - findet sich am häufigsten im mittleren Erwachsenenalter: So fallen die Anteile bei den 48- bis 53-Jährigen (13,6 Prozent) und den 54- bis 59-Jährigen am höchsten aus (15,2 Prozent). Das mag durch den Ende des mittleren Erwachsenenalters verstärkt einsetzenden Unterstützungsbedarf (vor allem seitens der Eltern) hervorgerufen werden bei gleichzeitig hoher Erwerbsbeteiligung bis etwa zum 60. Lebensjahr. Personen ab 60 Jahren sind deutlich seltener erwerbstätig (vgl. Kapitel 3) und entsprechend bewegt sich der Anteil derer mit Doppelverantwortung in dieser späten Erwerbsphase (60- bis 65-Jährigen) auf einem vergleichsweise geringen Niveau (7,3 Prozent).

\section{Frauen vereinbaren häufiger Erwerbstätigkeit und Unterstützungsaufgaben als Männer und Hochgebildete häufiger als niedriger Gebildete.}

Dabei zeigen sich Geschlechter- und Bildungsunterschiede (Abbildung 12-6): Während Frau- en und Männer im erwerbsfähigen Alter im Jahr 1996 noch gleich häufig Erwerbs- und Unterstützungsaufgaben miteinander vereinbart haben (6,9 Prozent beziehungsweise 7,2 Prozent), ist dieser Anteil vor allem unter den Frauen deutlich angestiegen. Zwar sind die Werte 2014 bei beiden Geschlechtern signifikant höher als 1996, aber der Zuwachs fällt bei Frauen deutlicher aus: Es zeigt sich eine Verdoppelung von 6,9 Prozent auf 13,9 Prozent. Es sind also zunehmend vor allem Frauen, die die Vereinbarkeitsfrage lösen müssen.

Das gilt auch für Unterstützungsleistende mit mittlerem und hohem Bildungsniveau: In beiden Gruppen lassen sich grundsätzlich höhere Anteile von Personen beobachten, die Unterstützung und Erwerbstätigkeit miteinander vereinbaren als unter Niedriggebildeten: Im Jahr 2014 sind das 11,7 Prozent (mittlere Bildung) beziehungsweise 12,8 Prozent (hohe Bildung) im Vergleich zu 5,1 Prozent (geringe Bildung). Abgesehen von leichten Rückgängen in den Zwischenjahren erweist sich der Anstieg zwischen 1996 und 2014 in allen Bildungsgruppen als signifikant. 


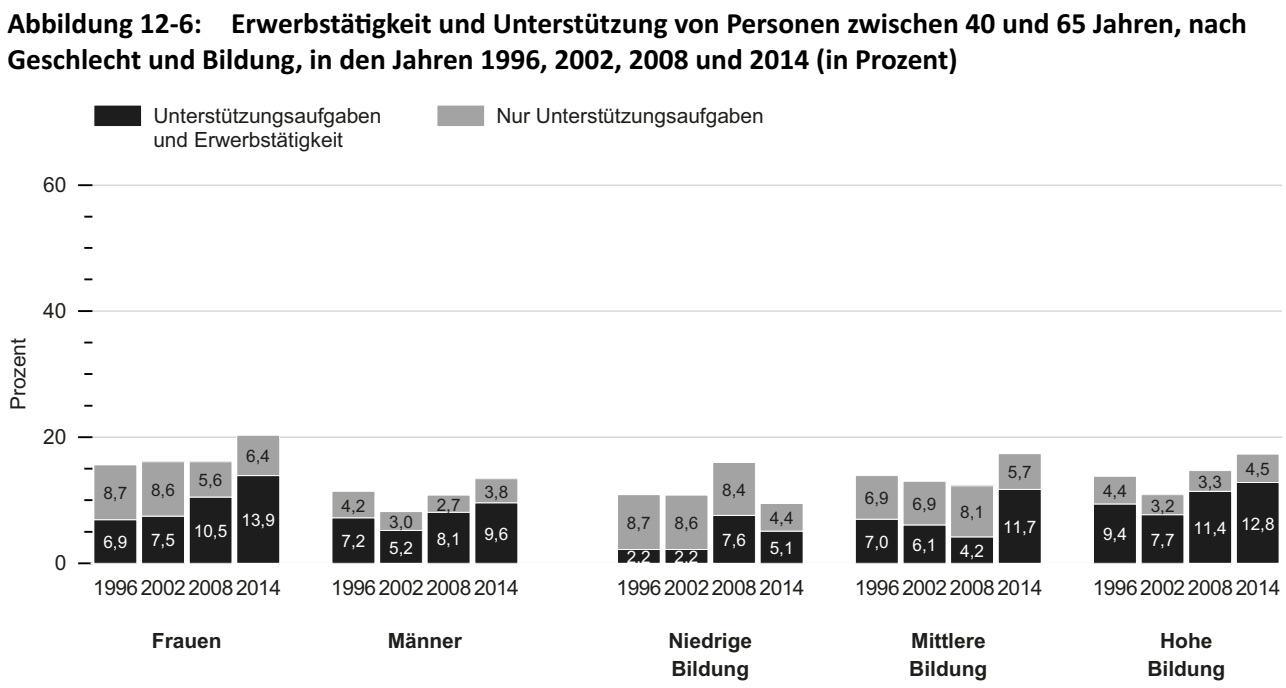

$\overline{\text { Quelle: DEAS } 1996}(\mathrm{n}=3.132), 2002(\mathrm{n}=1.834), 2008(\mathrm{n}=3.480), 2014(\mathrm{n}=3.526)$, gewichtet, gerundete Angaben; $(p<, 05)$.

Signifikante Unterschiede zwischen 1996 und 2014 für Männer und Frauen, sowie für alle drei Bildungsgruppen. Signifikant stärkere Zunahme zwischen 1996 und 2014 bei Frauen als bei Männern.

Es wurde eingangs die Frage aufgeworfen, ob sich die Zunahme später Geburten mittlerweile in einem Anstieg von Personen niederschlägt, die eine Mehrfachverantwortung haben. Um diese Frage zu beantworten, wird zusätzlich berücksichtigt, ob Sorge für mindestens ein minderjähriges Kind im Haushalt zu tragen ist (in Anlehnung an Künemund, 2002). Die Befunde belegen einen signifikanten Anstieg einer solchen Mehrfachverantwortung zwischen 1996 und 2014 (von 1,9 auf 3,2 Prozent; ohne Abbildung). Da neben der Erwerbsquote (vgl. Kapitel 3) und der Unterstützerquote (vorliegendes Kapitel) auch der Anteil der Personen zwischen 40 und 65 Jahren mit mindestens einem minderjährigen Kindern im eigenen Haushalt von 27 Prozent (1996) auf 31 Prozent (2014) leicht zugenommen hat (nicht signifikant), geht dieser Anstieg auf Verschiebungen in allen drei Bereichen zurück. Allerdings verdeutlichen die Zahlen auch, dass nach wie vor nur ein sehr geringer Anteil der erwerbsfähigen Bevölkerung ab dem 40. Lebensjahr hiervon betroffen ist. Auch ein Vergleich der empfundenen Belastung durch die geleistete Unterstützung erbringt keine signifikanten Unterschiede zwischen den unterschiedlichen Vereinbarkeitsgruppen: Erwerbstätige Personen im Alter zwischen 40 und 65 Jahren, die mindestens eine andere Person gesundheitsbedingt unterstützen, fühlen sich hiervon ebenso belastet wie nicht erwerbstätige Personen oder solche die zudem noch minderjährige Kinder im Haushalt betreuen.

\subsection{Diskussion und Implikationen}

Entgegen der Annahme einer rückläufigen informellen Unterstützung sowie einem Bedeutungsverlust der Kernfamilie bei der Erbringung dieser Versorgungsleistungen zeigt sich, dass 2014 mehr Personen in der zweiten Lebenshälfte Unterstützung für gesundheitlich eingeschränk- 
te Menschen leisten als 1996. Hierbei übernehmen nach wie vor Mitglieder des engsten Familienkreises die Hauptverantwortung. Das ist umso bemerkenswerter, weil gerade deren Verfügbarkeit abgenommen hat - sowohl mit Blick auf ihre Anzahl als auch ihre Gelegenheiten und Zeitressourcen, diese Aufgaben zu übernehmen. Unterstrichen wird das durch den steigenden Anteil von Personen in der zweiten Lebenshälfte, die gleichzeitig Unterstützung leisten und erwerbstätig sind. Das impliziert ein hohes Potenzial erwachsener Kinder und Partnerinnen beziehungsweise Partner, die gestiegene Nachfrage nach Unterstützung und Hilfe bei der Alltagsbewältigung zu decken.

Ähnlich der im Zusammenhang mit dem Ausbau wohlfahrtsstaatlicher Leistungen diskutierten Komplementaritätsthese (Daatland \& Herlofson 2003) dürften die Kapazitäten hierfür zum Teil durch Auslagerung zeit- und arbeitsintensiver (Pflege-)Tätigkeiten frei geworden sein. Es deutet sich eine stärker arbeitsteilige Organisation der nachgefragten Unterstützung zwischen mehreren Hilfeleistern und Pflegenden an. Die damit implizierte Entlastung der Familienangehörigen erlaubt es ihnen, sich stärker auf weniger anspruchsvolle Aufgaben konzentrieren zu können. Auch sollte sich damit der Druck einer möglichen Alleinverantwortung sowie Überforderung reduzieren. Ein Mix aus verschiedenen informellen und professionellen Hilfeleistern - im Sinne einer gemischten Verantwortung (Motel-Klingebiel, Tesch-Römer, \& von Kondratowitz 2005) - dürfte auch aus der Sicht der Empfänger positiv sein: So reduziert sich deren Abhängigkeit von einer Unterstützungsquelle und damit ihr Risiko eines Versorgungsdefizites bei (personellen) Ausfällen. Eine arbeitsteilige und dann möglicherweise auch spezialisierte Aufgabenübernahme kann zudem die Qualität der erhaltenen Hilfe und Pflege verbessern.

Nichtsdestotrotz legen aktuelle Entwicklungen zukünftige Versorgungsengpässe und Überlastungen nahe: So ist beispielsweise der deutliche Anstieg von Kinderlosigkeit derzeit noch nicht unter älteren Hilfs- und Pflegebedürftigen angekommen, wird aber in absehbarer Zukunft das Versorgungssystem vor ganze neue Her- ausforderungen stellen (vgl. Kapitel 14). Und auch die Erwerbstätigkeit unter älteren Frauen wird weiterhin ansteigen. Entsprechend ist eine Fortführung des Ausbaus von Maßnahmen zur Entlastung unterstützender und pflegender Angehöriger unabdinglich und dringlich: So sollten ambulante und professionelle Unterstützungsangebote, die ergänzend zur informellen Versorgung oder auch als Ersatz genutzt werden können, ausgebaut, breiter zugänglich und finanzierbar gemacht werden. Die Vereinbarkeit von Pflege- und Unterstützungsleistungen mit Erwerbstätigkeit ist weiterhin zu fördern, um sowohl eine finanzielle Mindestabsicherung für die Unterstützungspersonen sicherzustellen, als auch eine zeitliche Flexibilität zu schaffen, die die parallele Erledigung beider Aufgaben erleichtert. Diesbezüglich schlägt die jüngste Modifikation der Familienpflegezeit (2015) den richtigen Weg ein: Demnach wird für eine zehntägige Erwerbsfreistellung eine Lohnersatzleistung gewährt und es besteht ein Rechtsanspruch der Beschäftigten auf eine Erwerbsreduktion oder - unterbrechung bis zu 24 Monaten bei bestehendem Kündigungsschutz. Allerdings gelten viele dieser Regelungen nur für Angestellte in Betrieben mit einer Mindestgröße von 25 Mitarbeitern, womit ein großer Personenkreis ausgeschlossen bleibt. Das betrifft auch nichtverwandte Pflegepersonen. Sie werden bislang generell nicht von der Familienpflegezeit erfasst, bieten aber durchaus ein gewisses Potenzial für informelle Unterstützung (vgl. Kapitel 17).

Ebenso wäre es erstrebenswert, Männer und Niedriggebildete stärker einzubinden als bisher. Frauen und Personen mit mittlerem und hohem Bildungsniveau übernehmen diese Aufgaben nicht nur häufiger, sondern vereinbaren sie auch häufiger mit anderen Aufgabenbereichen - allen voran Erwerbstätigkeit. Insbesondere für Frauen, die bekanntermaßen auch die Hauptverantwortung für Kinderbetreuung und Haushaltsaufgaben übernehmen (vgl. Kapitel 13, 15), kann es zu einer Kumulation von Vereinbarkeitsanforderungen kommen - der es entgegenzuwirken gilt. Schließlich wäre die Bereitstellung von Leistungen bereits vor der Entstehung von Pflegebedarf insofern sinnvoll, da ein nicht unerheblicher Unterstützungsbedarf bereits 
unter dieser Grenze entsteht. Bilanzierend ist trotz sich abzeichnender Handlungsbedarfe zur Motivation und Unterstützung (potenziell) Hilfeleistender festzuhalten, dass ein beträchtlicher und wachsender Teil der Bevölkerung zwischen 40 und 85 Jahren Unterstützungsaufgaben für kranke und hilfebedürftige Angehörige übernimmt.

\section{Literatur}

Blinkert, B., \& Klie, T. (2008). Soziale Ungleichheit und Pflege. Aus Politik und Zeitgeschichte, 12(13), 25-33.

Blinkert, B., \& Klie, T. (2006). Die Zeiten der Pflege. Zeitschrift für Gerontologie und Geriatrie, 39(3), 202-210.

Cantor, M. H. (1979). Neighbors and friends: An overlooked resource in the informal support system. Research on Aging, 1(4), 434-463. doi: 10.1177/016402757914002.

Daatland, S. O., \& Herlofson, K. (2003). Families and welfare states: Substitution or complementary. In: A. Lowenstein \& J. Ogg (Hrsg.) OASIS: Old Age and Autonomy - The Role of Service Systems and Intergenerational Family Solidarity. (S. 281-305). Haifa: University of Haifa.

Deindl, C., Brandt, M., \& Hank, K. (2014). Generationen in Europa: Theoretische Perspektiven und empirische Befunde. In: A. Steinbach, M. Hennig \& O. Arránz Becker (Hrsg.) Familie im Fokus der Wissenschaft (Familienforschung, S. 299-325). Wiesbaden: Springer.

Dudel, C. (2015). Vorausberechnung des Pflegepotentials von erwachsenen Kindern für ihre pflegebedürftigen Eltern. Sozialer Fortschritt, 64(2), 14-26.

Forschungsgesellschaft für Gerontologie e.V. (2006). Wohnen im Alter. Dortmund: Institut Arbeit und Technik.

Geerts, J., \& Bosch, K. van den (2012). Transitions in formal and informal care utilisation amongst older Europeans: the impact of national contexts. European journal of ageing, 9(1), 27-37.

Geyer, J., \& Schulz, E. (2014). Who cares? Die Bedeutung der informellen Pflege durch Erwerbstätige in Deutschland. DIW Wochenbericht, 81(14), 294-301.

Haberkern, K., Schmid, T., \& Szydlik, M. (2015). Gender differences in intergenerational care in European welfare states. Ageing and society, 35(2), 298-320.

Haberkern, K., \& Szydlik, M. (2008). Pflege der Eltern ein europäischer Vergleich. Kölner Zeitschrift für Soziologie und Sozialpsychologie, 60(1), 82-105.

Heusinger, J. (2007). Freundin, Expertin oder Dienstmädchen - zu den Auswirkungen sozialer Ungleichheit auf die Funktion professioneller Pflegekräfte in häuslichen Pflegearrangements. Pflege \& Gesellschaft, 12(4), 318342.

Isfort, M. (2013). Anpassung des Pflegesektors zur Versorgung älterer Menschen. Aus Politik und Zeitgeschichte, 2013(4-5), 29-35.

Khan, H. T. A. (2014). Factors Associated with Intergenerational Social Support among Older Adults across the World. Ageing International, 39(4), 289-326. doi: 10.1007/s12126-013-9191-6.

Klaus, D. (2009). Why do adult children support their parents. Journal of Comparative Family Studies, 40, 227-241.

Klaus, D., \& Tesch-Römer, C. (2014). Pflegende Angehörige und Vereinbarkeit von Pflege und Beruf: Befunde aus dem Deutschen Alterssurvey 2008. DZA-Fact Sheet. Online: https://www.dza.de/fileadmin/dza/pdf/Fact_ Sheet_Pflege_Erwerbstaetigkeit_2014_09_12.pdf (zuletzt abgerufen 15.07.2016).

Kuhlmey, A., Dräger, D., Winter, M., \& Beikirch, E. (2010). COMPASS-Versichertenbefragung zu Erwartungen und Wünschen an eine qualitativ gute Pflege. Informationsdienst Altersfragen, 37(4), 4-10.

Künemund, H. (2002). Die "Sandwich-Generation"- typische Belastungskonstellation oder nur gelegentliche Kumulation von Erwerbstätigkeit, Pflege und Kinderbetreuung? Zeitschrift für Soziologie der Erziehung und Sozialisation, 22(4), 344-361.

Litwak, E. (1985). Helping the elderly: The complementary roles of informal networks and formal systems. New York: Guildford.

Motel-Klingebiel, A., Tesch-Römer, C., \& Kondratowitz, H.-J. von (2005). Welfare states do not crowd out the family: evidence for mixed responsibility from comparative analyses. Ageing and society, 25(6), 863-882.

Preuss, M. (2014). Vereinbarkeit von Pflege und Erwerbstätigkeit. Wiesbaden: Springer VS.

Schmid, T., Brandt, M., \& Haberkern, K. (2012). Gendered support to older parents: do welfare states matter? European journal of ageing, 9(1), 39-50. 
Statistisches Bundesamt (2015b). Pflegestatistik 2013. Wiesbaden: Statistisches Bundesamt.

Stüben, C., \& Schwanenflügel, M. von (2015). Vereinbarkeit von Familie, Pflege und Beruf: Die neuen gesetzlichen Regelungen als Unterstützung für Pflegebedürftige und Angehörige. Gesundheits- und Sozialpolitik, 69(1), 76-79.
TNS Infratest Sozialforschung (2011). Abschlussbericht zur Studie "Wirkungen des Pflege-Weiterentwicklungsgesetzes". Berlin: Bundesministerium für Gesundheit.

Wetzstein, M., Rommel, A., \& Lange, C. (2015). Pflegende Angehörige - Deutschlands größter Pflegedienst. GBE Kompakt, 6(3), 3-11.

Open Access Dieses Kapitel wird unter der Creative Commons Namensnennung 2.5 International Lizenz (http://creativecommons.org/licenses/by/2.5/deed.de) veröffentlicht, welche die Nutzung, Vervielfältigung, Bearbeitung, Verbreitung und Wiedergabe in jeglichem Medium und Format erlaubt, sofern Sie den/die ursprünglichen Autor(en) und die Quelle ordnungsgemäß nennen, einen Link zur Creative Commons Lizenz beifügen und angeben, ob Änderungen vorgenommen wurden.

Die in diesem Kapitel enthaltenen Bilder und sonstiges Drittmaterial unterliegen ebenfalls der genannten Creative Commons Lizenz, sofern sich aus der Abbildungslegende nichts anderes ergibt. Sofern das betreffende Material nicht unter der genannten Creative Commons Lizenz steht und die betreffende Handlung nicht nach gesetzlichen Vorschriften erlaubt ist, ist für die oben aufgeführten Weiterverwendungen des Materials die Einwilligung des jeweiligen Rechteinhabers einzuholen. 